INPLASY

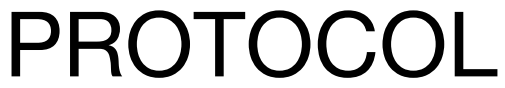

To cite: Cheng et al.

Effectiveness and safety of Liuhedan for treating cellulitis

A protocol for a systematic review and meta-analysis. Inplasy protocol 202110049. doi:

10.37766/inplasy2021.1.0049

Received: 14 January 2021

Published: 15 January 2021

Corresponding author:

Cheng Tao

hxjz_CT1989@163.com

Author Affiliation:

West China Hospital

Support: No. $2018 S Z 0390$.

Review Stage at time of this submission: The review has not yet started.

Conflicts of interest: None.

\section{Effectiveness and safety of Liuhedan for treating cellulitis A protocol for a systematic review and meta-analysis}

Cheng, T1; Liu, BF2; Han, TY3; Gu, ZH; Pan, P5; Yu, HF6.

Review question / Objective: P: Patients with cellulitis; I: Treat with Liuhedan; C: Treat without Liuhedan; O: duration of treating with antibiotic, duration of hospital stays and hospital mortality; S: RCT.

Condition being studied: Liuhedan is used to treat cellulitis in China. But at present, there is no systematic evaluation report on its therapeutic effectiveness and safety. This protocol aims to reveal the efficacy and safety of Liuhedan for treating cellulitis.

Information sources: We will search the EMBASE, Wanfang, Web of Knowledge, Weipu, PubMed, Zhiwang, ClinicalTrials.gov and Cochrane Library from inception to Mar 2021 to retrieve relevant studies. We will also search citations of relevant primary and review. Authors of abstract in the meeting will be further searched in PubMed for potential full articles. To minimize the risk of publication bias, we will conduct a comprehensive search that included strategies to find published and unpublished studies.

INPLASY registration number: This protocol was registered with the International Platform of Registered Systematic Review and Meta-Analysis Protocols (INPLASY) on 15 January 2021 and was last updated on 15 January 2021 (registration number INPLASY202110049).

\section{INTRODUCTION}

Review question / Objective: P: Patients with cellulitis; I: Treat with Liuhedan; C: Treat without Liuhedan; $\mathrm{O}$ : duration of treating with antibiotic, duration of hospital stays and hospital mortality; S: RCT.
Condition being studied: Liuhedan is used to treat cellulitis in China. But at present, there is no systematic evaluation report on its therapeutic effectiveness and safety. This protocol aims to reveal the efficacy 
and safety of Liuhedan for treating cellulitis.

\section{METHODS}

Participant or population: Age $\geq 18$ years old and diagnosis of cellulitis.

Intervention: Treated with Liuhedan.

Comparator: Treated without Liuhedan.

Study designs to be included: RCT.

Eligibility criteria: The inclusion criteria for the study will include: (1) studies with patient age $\geq 18$ years old and diagnosis of cellulitis; (2) conference abstracts were only included when they provided adequate relevant information for assessment; (3) the patients with cellulitis was divided into two groups (treated with Liuhedan or without Liuhedan).

Information sources: We will search the EMBASE, Wanfang, Web of Knowledge, Weipu, PubMed, Zhiwang, ClinicalTrials.gov and Cochrane Library from inception to Mar 2021 to retrieve relevant studies. We will also search citations of relevant primary and review. Authors of abstract in the meeting will be further searched in PubMed for potential full articles. To minimize the risk of publication bias, we will conduct a comprehensive search that included strategies to find published and unpublished studies.

Main outcome(s): Hospital mortality.

Additional outcome(s): duration of treating with antibiotic and duration of hospital stays.

Quality assessment / Risk of bias analysis: Risk of bias assessment will be carried out according to the Newcastle-Ottawa Scale (NOS) to rate the internal validity of the individual studies, and funnel plots will be constructed to assess the risk of publication bias.

Strategy of data synthesis: All pairwise meta-analytic calculations will be performed with Review Manager software (RevMan) version 5.3 (Cochrane Collaboration). Heterogeneity will be examined by computing the $Q$ statistic and $I^{2}$ statistic, and presence of reporting bias by visual inspection of funnel plots. Statistical significance was considered when the $P$ value $<0.05$.

Subgroup analysis: Patients with cellulitis treated with Liuhedan or without Liuhedan.

Sensibility analysis: Heterogeneity will be examined by computing the $Q$ statistic and $I^{2}$ statistic, and presence of reporting bias by visual inspection of funnel plots. Statistical significance was considered when the $P$ value $<0.05$.

Country(ies) involved: China.

Keywords: patients; Liuhedan; Traditional Chinese medicine; prognosis; mortality.

Contributions of each author:

Author 1 - Cheng Tao.

Email: hxjz_CT1989@163.com

Author 2 - Bo-Fu Liu.

Email: 837718077@qq.com

Author 3 - Tian-Yong Han.

Email:654191633@qq.com

Author 4 - Gu Zhi-Han.

Email: 18908337354@163.com

Author 5 - Pan Pan.

Email: 2210670239@qq.com

Author 6 - Yu Haifang.

Email: yuhaifang@wchscu.cn 